\title{
Los desafíos éticos del periodismo en la era del big data: análisis de códigos deontológicos latinoamericanos
}

\section{Jesús Díaz del Campo-Lozano' María-Ángeles Chaparro-Domínguez²}

Recibido: 2017-07-11

Enviado a pares: 2017-07-25
Aprobado por pares: 2017-09-13

Aceptado: 2017-10-04

DOI: 10.5294/pacla.2018.21.4.8

Para citar este artículo / to reference this article / para citar este artigo

Díaz del Campo-Lozano, J. y Chaparro-Domínguez, M. A. (2018). Los desafíos éticos del periodismo en la era del big data: análisis de códigos deontológicos latinoamericanos. Palabra Clave, 21(4), 1136-1163. doi: 10.5294/pacla.2018.21.4.8

\section{Resumen}

El periodismo de investigación, clave en el buen funcionamiento de cualquier sociedad democrática, se enfrenta a numerosos desafíos éticos. La introducción de las técnicas del big data ha dado lugar al periodismo de datos, que supone un nuevo reto en este sentido. El principal objetivo de este estudio es analizar la existencia de pautas deontológicas sobre periodismo y big data en un área geográfica que hasta ahora no se ha estudiado en ese ámbito: América Latina. Para ello, se emplea la metodología del análisis de contenido sobre una selección de 18 códigos éticos de asociaciones de periodistas de 18 países para comprobar si cumplen con los principios establecidos por autores como Bradshaw, Ward, Singer, Dörr y Hollnbuchner, quienes adaptan la deontología periodística al contexto concreto del big data. Los resultados muestran que los códigos están poco adaptados a

1 orcid.org/0000-0001-5014-8749. Universidad Internacional de La Rioja, España. jesus.diaz@unir.net

2 orcid.org/0000-0001-7571-388X. Universidad Internacional de La Rioja, España.

marian.chaparrodominguez@unir.net 
los principios éticos relacionados con la verificación de datos. En cambio, sí que están en la línea de lo que se considera objetivo periodístico (objetividad, honestidad y servicio público) en el contexto del periodismo de datos. Además, se observa que, en general, los códigos mejor adaptados son los que se han aprobado o modificado en los últimos años, como son, entre otros, los de Uruguay, Colombia y Chile. Se concluye que sería recomendable revisar los códigos más antiguos y redactar códigos o recomendaciones éticas específicas referidas al big data, además de actualizar el código supranacional de referencia en América Latina, el Código Latinoamericano de Ética Periodística, que data de 1979.

\section{Palabras clave}

Ética de los medios de comunicación; códigos éticos; América Latina; periodistas; medios de comunicación; medios de comunicación de masas (Fuente: Tesauro de la Unesco). 


\section{Ethical Challenges of Journalism in the Era of Big Data: An Analysis of Latin American Deontological Codes}

\section{Abstract}

Investigative journalism, which is crucial to the proper functioning of any democratic society, faces numerous ethical challenges. The introduction of big data techniques has given rise to data journalism that presumes a new challenge in this sense. The main objective of the study is to analyze the existence of deontological guidelines on journalism and big data in Latin America, geographical area that has not been studied in that respect until now. To do so, content analysis was the methodology applied to a selection of 18 ethical codes adopted by associations of journalists in 18 countries to check if they comply with the principles established by authors such as Bradshaw, Ward, Singer, Dörr and Hollnbuchner, who adapt journalistic deontology to the specific context of big data. The results show the codes are poorly adapted to the ethical principles related to data verification. Instead, they are in line with what is considered to be a journalistic target (objectivity, honesty and public service) in the context of data journalism. It is also observed that, in general, the best adapted codes are those that have been approved or modified in recent years, such as the ones in Uruguay, Colombia and Chile, among others. It is concluded that it would be advisable to review the oldest codes and write specific codes or recommendations referring to big data, in addition to updating the supranational code of reference in Latin America: the Latin American Code of Journalistic Ethics, which dates from 1979.

\section{Keywords}

Ethics of the media; ethical codes; Latin America; journalists; mass media (Source: Unesco Thesaurus). 


\section{Os desafios éticos do jornalismo na era do big data: análise de códigos deontológicos latino-americanos}

\section{Resumo}

O jornalismo investigativo, fundamental para o bom funcionamento de qualquer sociedade democrática, enfrenta inúmeros desafios éticos. A introdução das técnicas do big data dão lugar ao jornalismo de dados, que supõe um novo obstáculo nesse sentido. O principal objetivo deste estudo é analisar a existência de pautas deontológicas sobre jornalismo e big data numa área geográfica que até agora não foi estudada nesse âmbito: a América Latina. Para isso, utiliza a metodologia da análise de conteúdo sobre uma seleção de 18 códigos éticos de associações de jornalistas de 18 países para comprovar se cumprem com os princípios estabelecidos por autores como Bradshaw, Ward, Singer, Dörr e Hollnbuchner, os quais adaptam a deontologia jornalística ao contexto concreto do big data. Os resultados mostram que os códigos estão pouco adaptados aos princípios éticos relacionados com a verificação dos dados. Em compensação, estão na linha do que se considera objetivo jornalístico (objetividade, honestidade e serviço público) no contexto do jornalismo de dados. Além disso, observa-se que, em geral, os códigos mais bem adaptados são os que têm sido aprovados ou modificados nos últimos anos, como são, entre outros, os do Chile, da Colômbia e do Uruguai. Conclui-se que seria recomendável revisar os códigos mais antigos e redigir códigos ou recomendações específicas referentes ao big data, além de atualizar o código supranacional de referência na América Latina, o Código Latino-americano de Ética Jornalística, que data de 1979.

\section{Palavras-chave}

Ética dos meios de comunicação, códigos éticos, América Latina, jornalistas, meios de comunicação (Fonte: Tesauro da Unesco). 


\section{Introducción}

\section{El big data y el periodismo de datos en el nuevo sistema mediático}

El periodismo asiste a un profundo proceso de reconversión marcado, entre otras cosas, por el entorno digital (Casero-Ripollés, 2012) que ha dado lugar a nuevos modelos de negocio, nuevas técnicas y nuevas especialidades. Una de ellas es el llamado periodismo de datos, cuya filosofía se basa en el empleo de las técnicas propias del big data para encontrar historias que resulten de interés para la sociedad. Se trata, pues, de una propuesta periodística que surge de la web y se caracteriza por el rigor metodológico de las ciencias sociales a la hora de extraer, sistematizar y tratar datos que después serán empleados para publicar historias periodísticas (La-Larosa y Sandoval-Martín, 2016).

El big data puede ofrecer una mirada fresca y percepciones nuevas, porque no está comprometido por el pensamiento convencional y los sesgos inherentes propios de cada campo (Mayer-Shönberger y Cukier, 2013). Una de sus posibilidades es descubrir historias de interés social gracias a la explotación estadística de grandes bases de datos que dejan a un lado condicionantes tradicionales teóricos o normativos (Anderson, 2008).

Sin embargo, como advierte Bruns (2016), esta nueva disciplina también implica diversas amenazas para las buenas prácticas periodísticas, como la búsqueda de obtener las mejores métricas y la mayor popularidad en detrimento de la calidad del producto. Por ello, cada vez son más los medios que incluyen un apartado específico dedicado a esta modalidad periodística en sus normas de estilo, como la agencia de noticias Associated Press, que lo ha incorporado en su edición de 2017.

A pesar de que realmente el manejo de datos ha sido una constante en la labor de los periodistas, esta nueva modalidad implica, por un lado, el acceso a grandes cantidades de datos, y por otro, el empleo de nuevas herramientas para la búsqueda, extracción, análisis y visualización de estos (Crucianelli, 2012; Gray, Chambers y Bounegru, 2012; Parasie, 2015; Perry y 
Paz, 2014). Su origen histórico se encuentra en el llamado periodismo de precisión, que comenzó a desarrollar el periodista Philip Meyer a mediados del siglo XX y que combinaba las técnicas periodísticas con otras propias de otras disciplinas, como la sociología, la estadística y la informática (Chaparro, 2014; Dader, 1997). Por tanto, puede decirse que el periodismo de datos supone una evolución que da continuidad al periodismo de precisión (Elías-Pérez, 2015).

La investigación académica ha empleado otros conceptos como periodismo asistido por ordenador o periodismo computacional (Anderson, 2012; Carlson, 2015; Diakopoulos, 2015; Kent, 2015), para referirse a prácticas que comparten elementos con el periodismo de datos. Según Coddington (2015), el periodismo asistido por ordenador recurre a métodos propios de ciencias sociales para realizar proyectos de investigación relativos a asuntos públicos, mientras que el periodismo de datos se caracteriza por su carácter híbrido y su mayor apertura a la participación del público. Por último, el periodismo computacional se centra en los procesos de automatización de la información.

Al mismo tiempo, esta nueva modalidad da lugar a nuevos perfiles de periodistas, cuyas características va definiendo sobre la marcha la propia práctica profesional (Fink y Anderson, 2015; López-García, TouralBran y Rodríguez-Vázquez, 2016), y que pasan, entre otros elementos, por el empleo óptimo de software especializado, la correcta gestión de bases de datos y la habilidad para presentar la información mediante nuevos formatos, en los que los mapas y las visualizaciones desempeñan un papel fundamental (Ferreras, 2013).

\section{Los desafíos éticos del periodismo de datos actual}

La nueva realidad del periodismo digital genera multitud de desafíos éticos que afectan, además, cuestiones capitales en la labor periodística, como la verificación de la información ante la sobreabundancia de esta (García-Avilés, 2014), la interacción entre profesionales y usuarios (Suárez Villegas, 2015), la relación con las fuentes (Deuze y Yeshua, 2001), la imparcialidad (Ess, 2009) o las implicaciones respecto de la privacidad (Lewis y Westlund, 2015). 
Son cuestiones que no resultan novedosas en el día a día del profesional de la información, pero cuya naturaleza se ve redefinida en el escenario de internet y las TIC (tecnologías de la información y la comunicación) (Deuze y Yeshua, 2001). Se trata, por tanto, de un nuevo escenario que requiere una redefinición de muchos de los principios éticos tradicionales del periodismo (Díaz-Campo y Segado-Boj, 2014, 2015; Hayes, Singer y Ceppos, 2007; Pavlik, 2001).

En este sentido, el fenómeno del big data trae consigo sus propios retos éticos, que merecen ser estudiados a medida que los periodistas se van enfrentando a ellos (Lewis y Westlund, 2015). Estos conflictos se refieren a la privacidad, la seguridad de la información o la manipulación de los datos, entre otros (Crawford, Miltner y Gray, 2014; Suárez-Gonzalo, 2017). Por tanto, este nuevo periodismo exige igualmente un nuevo planteamiento en términos éticos, ya que, entre otras cosas, el mero hecho de que los datos con los que se trabaja sean cuantificables no supone de manera automática que equivalgan a la verdad absoluta (Boyd y Crawford, 2012), en especial si esos datos no se contextualizan adecuadamente (McBride, 2016).

Son ya varios los autores que han abordado la tarea de intentar definir una serie de principios éticos fundamentales para el periodismo de datos. Fairfield y Shtein (2014) plantean un marco basado en las obligaciones $\mathrm{y}$ se inspiran en algunos de los principios éticos fundamentales que rigen cualquier investigación científica que implique la presencia de seres humanos, como la autonomía, la beneficencia o la justicia para, a partir de ahí, extraer tres cuestiones más específicas del periodismo de datos: el tamaño de la muestra, el que el conjunto de los datos constituya un todo homogéneo y no una mera acumulación de estos y los posibles perjuicios y daños que se puedan causar cuando se difunda la información.

Por su parte, Bradshaw (2014) apunta a aspectos como el rigor a la hora de reunir los datos, la importancia de contextualizarlos adecuadamente, el desafío de la interactividad con el público, la necesidad de ser algo escépticos con los datos provenientes de encuestas realizadas en línea, la privacidad o la protección de las fuentes, entre otras cuestiones. Mientras 
Craig, Ketterer y Yousuf (2017) presentan un enfoque basado en marcos genéricos de actuación y, en buena medida, en la ética deontológica, que se concreta en seis categorías básicas: 1) libertad frente a responsabilidad, 2) propósito periodístico, 3) privacidad, 4) verificación, 5) consecuencias y 6) alternativas.

Finalmente, Dörr y Hollnbuchner (2017) formulan la que probablemente constituye la aproximación más ambiciosa y exhaustiva a ese objetivo de plantear un marco ético de actuación para el periodismo de datos, ya que estructura las diferentes cuestiones éticas que entran en juego en tres esferas: 1) organizacional, 2) profesional y 3) social; y por otro lado, en tres etapas del proceso de producción de la información: 1) recogida de esta, 2) proceso de elaboración y 3 ) difusión.

Como resultado de todo ello, señalan los siguientes elementos de interés ético:

1. En el proceso de recogida de información:

a. Respecto de los datos: fiabilidad, respeto por la privacidad, empleo de métodos apropiados para conseguir los datos, posibles prejuicios, derechos y autoría de los datos e implicaciones económicas.

b. Respecto de la actuación del periodista: transparencia, objetividad, responsabilidad, exactitud, posibles prejuicios, autoría y derechos del software o técnicas empleadas e implicaciones éticas.

2. En la elaboración de la información: control y comprobación del software y de las técnicas empleadas.

3. A la hora de difundir la información: autoría de los resultados, transparencia (revelación de la fuente de la información y de los métodos o sistemas informáticos en el caso de que se hayan empleado), adecuación a las normas legales y monitorización externa de esos resultados.

Por tanto, el elenco de cuestiones éticas en juego parece lo suficientemente amplio como para plantearse abordarlas de manera específica. Pues bien, la vía más habitual para que los periodistas lleven a la práctica estos principios es materializarlos a través de los distintos mecanismos de 
autorregulación, que suponen una especie de vía intermedia entre la ética y el derecho (Suárez-Villegas, 1999). Estos mecanismos abarcan diferentes iniciativas, acuerdos, organismos e instituciones, cuyo objetivo es hacer efectivos esos principios y tienen como característica fundamental haber sido impulsados por los propios periodistas (Aznar, 2005, 2011). De entre todos ellos, suelen tener una especial importancia los códigos deontológicos, que pueden definirse como documentos que contienen una serie de criterios y pautas deontológicos que quienes ejercen una profesión consideran básicas (Díaz-Campo, 2016).

En consecuencia, el análisis de los distintos mecanismos de autorregulación y, en particular, de los códigos puede constituir una vía para comprobar en qué medida los periodistas de datos disponen de unas pautas claras para llevar a cabo su labor de una manera rigurosa y responsable. Sin embargo, el único antecedente localizado al realizar esta investigación es el llevado a cabo por Díaz-Campo, Chaparro-Domínguez y Segado-Boj (2016) al analizar distintos mecanismos de autorregulación en España. Su conclusión final es que el periodismo de datos se halla escasamente regulado en ese país, debido, entre otras cosas, a que la naturaleza y redacción concreta de los principios están más orientadas al periodismo en general, de forma que no se da una adecuada respuesta a los desafíos que surgen en esta especialidad concreta.

De ahí que el objetivo de esta investigación sea extender ese análisis a un ámbito geográfico particularmente relevante en el campo del periodismo en general y de la ética en particular por las singularidades que presenta (López-Talavera, 1998; Díaz-Campo, 2004; Oller y Barredo, 2013; Parra Valcarce, Real Rodríguez y López Talavera, 2017). Se trata de América Latina, cuyo primer antecedente de código deontológico se sitúa en Brasil en 1923, con la Declaración de São Paulo, a la que siguieron otras iniciativas nacionales en Cuba, Perú, Argentina, Venezuela o Chile, y otras de tipo supranacional (López-Talavera, 1998).

Pero, sin duda, un acontecimiento que supuso un antes y un después en la ética periodística de América Latina fue la celebración del II Congreso 
de la Federación Latinoamericana de Periodistas en 1979, dentro del cual se aprobó el Código Latinoamericano de Ética Periodística, en cuya elaboración se invirtieron tres años y que en buena medida ha servido como referencia para los códigos nacionales de ese ámbito geográfico. Los diferentes análisis llevados a cabo sobre estos códigos y referidos al periodismo en general han recogido, por un lado, algunas particularidades en cuanto a su contenido concreto, y por otro, una necesidad de revisión para adaptarse a la nueva realidad periodística (Díaz-Campo, 2004; López-Talavera, 1998).

Por tanto, el objetivo de este trabajo es analizar los códigos de ética periodística actualmente vigentes en América Latina para detectar cuál es el grado de presencia de principios éticos que hagan referencia al periodismo de datos y, por tanto, averiguar hasta qué punto está regulada en ellos esta especialidad.

\section{Metodología}

Para alcanzar el objetivo señalado, se ha realizado un análisis de contenido cualitativo de los códigos deontológicos de las principales asociaciones y colegios de periodistas de los países de América Latina. ${ }^{3}$ Partiendo del contenido de estos códigos, se ha escogido esta metodología tomando como referente a Craig, Ketterer y Yosuf (2017), quienes la utilizaron en un estudio sobre las dimensiones éticas de un reportaje de datos publicado en el medio Journal News de Nueva York (Estados Unidos) (Spones, 19 enero 2013) y su posterior discusión en el seno del Instituto Nacional para el Periodismo Asistido por Ordenador (The National Institute for Computer-Assisted Reporting).

Este estudio se basa en sus frames generales y se completa con una serie de categorías específicas, tomadas de diversos autores que han abordado el surgimiento de nuevos desafíos éticos en el ámbito del periodismo digital y de datos: Dörr y Hollnbuchner (2017), Singer (2007), Ward (2010), Bradshaw (2014), Díaz-Campo y Segado-Boj (2015), Suárez-

3 No se ha incluido el código de Nicaragua, porque todavía no se ha aprobado. En 2008, comenzaron a trabajar en su creación el Colegio de Periodistas de Nicaragua, la Unión de Periodistas de Nicaragua y la Asociación de Periodistas de Nicaragua, pero no llegaron a terminarlo y aprobarlo. 
Gonzalo (2017), Crawford, Miltner y Gray (2014). De este modo, se ha confeccionado un código de análisis de contenido cualitativo que recoge y unifica las principales aportaciones teóricas del campo de estudio en los últimos años (tabla 1).

\section{Tabla 1. Esquema general del código de análisis cualitativo de la investigación}

\begin{tabular}{|c|c|}
\hline Frames & \\
\hline $\begin{array}{c}\text { Craig, Ketterer y Yosuf } \\
(2017)\end{array}$ & Categorías \\
\hline \multirow{3}{*}{$\begin{array}{l}\text { Libertad vs. } \\
\text { responsabilidad }\end{array}$} & Métodos apropiados en la recolección de los datos (Dörr y Hollnbuchner, 2017) \\
\hline & Transparencia (Singer, 2007; Ward, 2010) \\
\hline & Rigor (Singer, 2007; Ward 2010) \\
\hline \multirow{3}{*}{ Objetivo periodístico } & Servicio público (Singer, 2007; Ward 2010) \\
\hline & Objetividad (Dörr y Hollnbuchner, 2017) \\
\hline & Honestidad (Singer, 2007; Ward 2010) \\
\hline \multirow{2}{*}{ Privacidad } & Respeto por la privacidad (Dörr y Hollnbuchner, 2017) \\
\hline & Seguridad de la información (Suárez-Gonzalo, 2017) \\
\hline \multirow{5}{*}{ Verificación } & Confirmación y análisis de los datos (Dörr y Hollnbuchner, 2017) \\
\hline & Evitar la manipulación de los datos (Crawford, Miltner y Gray, 2014) \\
\hline & Contextualización de los datos (Bradshaw, 2014) \\
\hline & Fiabilidad de los enlaces e hipervínculos (Díaz-Campo y Segado-Boj, 2015) \\
\hline & Control y comprobación del software y de las técnicas empleadas (Dörr y Hollnbuchner, 2017) \\
\hline \multirow{4}{*}{ Consecuencias } & Protección de las fuentes (Bradshaw, 2014) \\
\hline & Interactividad con el público (Bradshaw, 2014) \\
\hline & Autoría de los datos y los resultados (Dörr y Hollnbuchner, 2017) \\
\hline & Adecuación a las normas legales (Dörr y Hollnbuchner, 2017) \\
\hline
\end{tabular}

Fuente: elaboración propia.

Se ha analizado la presencia de principios éticos relacionados con el periodismo de datos en los códigos deontológicos de las principales asociaciones de periodistas de dieciocho países de América Latina. Se han excluido del análisis los códigos de carácter regional o supranacional, y en aquellos países en los que existe más de un documento de referencia de este tipo, se ha seleccionado el que se considera más representativo en cada caso.

A continuación, se recogen los códigos y el año de creación o modificación del documento (en el anexo 1 pueden verse más detalles de todos ellos): 
- Argentina: Código de Ética de la Prensa Argentina (2012)

- Bolivia: Código de Ética (1991)

- Brasil: Código de Ética y Autorregulación (1991)

- Chile: Código de Ética (2015)

- Colombia: Código de Ética (2006)

- Costa Rica: Código de ética de las y los profesionales en comunicación (2011)

- Cuba: Código de Ética del Periodista (2013)

- Ecuador: Código de Ética Profesional del Periodista (1980)

- El Salvador: Código de ética para los periodistas y profesionales de la comunicación en El Salvador (2012)

- Guatemala: Código de Ética (2000)

- Honduras: Código de Ética (1979)

- México: Código de Ética (1996)

- Panamá: Código de Ética Periodística (2007)

- Paraguay: Código de Ética (1999)

- Perú: Código de Ética (1988)

- República Dominicana: Código de Ética (1994)

- Uruguay: Código de Ética Periodística (2013)

- Venezuela: Código de Ética del Periodista Venezolano (2013)

Con el fin de validar el código de análisis cualitativo, se llevó a cabo un pretest con dos de los códigos del análisis (uno de la década de 1980 y otro reciente para que la prueba fuese más completa). Fruto de ese pretest se reformularon algunas de las categorías enunciadas para que se adaptasen correctamente al objeto de análisis. 


\section{Resultados}

De los cinco marcos generales estudiados, el que tiene mayor presencia en los códigos es el objetivo periodístico, ya que $72.2 \%$ de los textos analizados recoge alguno de sus principios (objetividad, honestidad y servicio público). En el extremo opuesto, se sitúa el frame de verificación, ya que solo 27.8 \% de los códigos incorpora alguno de sus principios (contextualización de los datos o fiabilidad de los enlaces, por ejemplo).

Dejando a un lado los frames generales, en el nivel de los principios éticos, se observa cómo el más frecuente es el de servicio público, presente en todos los códigos analizados (tabla 2). La protección a las fuentes y el respeto a la privacidad son otros principios populares, con 88.9 y $83.3 \%$ de presencia, respectivamente. Por el contrario, los principios de seguridad de la información, fiabilidad de los enlaces e hipervínculos y el control

\section{Tabla 2. Principios éticos presentes en los códigos latinoamericanos analizados}

\begin{tabular}{|l|c|}
\hline \multicolumn{1}{|c|}{ Principios } & Frecuencia (\%) y números de códigos \\
\hline Servicio público & $100(18)$ \\
\hline Protección de las fuentes & $88.9(16)$ \\
\hline Respeto por la privacidad & $83.3(15)$ \\
\hline Adecuación a las normas legales & $77.8(14)$ \\
\hline Objetividad & $72, .2(13)$ \\
\hline Autoría de los datos y los resultados & $66.7(12)$ \\
\hline Rigor & $61.1(11)$ \\
\hline Confirmación y análisis de los datos & $50(9)$ \\
\hline Evitar la manipulación de los datos & $50(9)$ \\
\hline Métodos apropiados para la recolección de datos & $50(9)$ \\
\hline Honestidad & $44.4(8)$ \\
\hline Contextualización de los datos & $38.9(7)$ \\
\hline Interactividad con el público & $27.8(5)$ \\
\hline Transparencia & $16.7(3)$ \\
\hline Control y comprobación del software y de las técnicas empleadas & $0(0)$ \\
\hline Fiabilidad de los enlaces e hipervínculos & $0(0)$ \\
\hline Seguridad de la información & $0(0)$ \\
\hline
\end{tabular}

Fuente: elaboración propia. 
y la comprobación del software y de las técnicas empleadas no han aparecido recogidos en ninguno de los códigos estudiados. El principio de seguridad de la información se refiere a que los datos que cualquier persona haya proporcionado con carácter privado estén realmente seguros y no al alcance de cualquiera (Suárez-Gonzalo, 2017). En cuanto a los enlaces e hipervínculos, se trata de que los sitios a los que enlace un medio sean fiables y cumplan igualmente las normas éticas básicas (Díaz-Campo y Segado-Boj, 2015). Con respecto al control y la comprobación del software y las técnicas empleadas, el periodista ha de preguntarse si existe algún tipo de control humano en el trabajo periodístico desarrollado por un programa informático, como puede ser la extracción de información de grandes bases de datos (Dörr y Hollnbuchner, 2017).

En cuanto a países, se observa que los códigos mejor adaptados al periodismo de datos son los de Uruguay, Colombia y Chile, que se han aprobado o modificado en los últimos años (2013, 2006 y 2015, respectivamente) (tabla 3). Los códigos de las asociaciones de periodistas de Perú (de 1988) y de México (de 1996) son los que incluyen un menor número de principios relacionados con el periodismo y el big data.

Asimismo, el análisis de la redacción específica de cada principio en los códigos revela que, en la mayoría de los casos, se trata de directrices orientadas al periodismo general, pero que resultan perfectamente aplicables al big data. Por el contrario, las recomendaciones más orientadas al periodismo digital son más escasas. De hecho, solo dos países, Chile y Uruguay, contienen un apartado específico sobre periodismo digital. A continuación, se ahonda en esta cuestión recogiendo las normas más directamente relacionadas con las tecnologías de la información y la comunicación que se han localizado en el análisis para cada uno de los marcos o frames establecidos.

\section{Libertad vs. responsabilidad}

Trece de los dieciocho códigos analizados $(72.2 \%)$ contienen al menos alguna referencia a este marco. El principio ético concreto al que se presta más atención es el rigor, que constituye, al mismo tiempo, el único en el que aparecen menciones explícitas a las TIC. Así, por ejemplo, el código 
de Chile señala en su artículo 10: "El o la periodista que utilice material informativo desde la Red está obligado a citar el origen del mismo. Es decir, el periodista debe actuar con el mismo rigor que aplica en las fuentes de información en otras plataformas”. Por su parte, el código de Uruguay establece en su artículo 47: "En caso de reproducir contenidos tomados de redes sociales los periodistas deben proceder con el mismo rigor que aplican con el resto de las fuentes de información".

\section{Tabla 3. Contenido de los códigos periodísticos latinoamericanos}

\begin{tabular}{|c|c|c|c|c|c|c|c|c|c|c|c|c|c|c|c|c|c|c|}
\hline Países* & I & II & III & IV & $\mathbf{v}$ & VI & VII & VIII & IX & $\mathbf{x}$ & XI & XII & XIII & XIV & $\mathbf{X V}$ & XVI & XVII & Total \\
\hline Argentina (2012) & $\mathrm{X}$ & & $\mathrm{X}$ & $\mathrm{x}$ & & $\mathrm{X}$ & $\mathrm{X}$ & & $\mathrm{X}$ & & & & & & $\mathrm{X}$ & & $\mathrm{X}$ & 8 \\
\hline Bolivia (1991) & $\mathrm{X}$ & & & $\mathrm{X}$ & & $\mathrm{x}$ & $\mathrm{X}$ & & & & & & & & & $\mathrm{X}$ & $\mathrm{X}$ & 6 \\
\hline Brasil (1991) & & & & $\mathrm{X}$ & $\mathrm{x}$ & & $\mathrm{X}$ & & & & $\mathrm{X}$ & & & $\mathrm{x}$ & & & $\mathrm{X}$ & 6 \\
\hline Chile (2015) & $\mathrm{X}$ & $\mathrm{x}$ & $\mathrm{X}$ & $\mathrm{X}$ & $\mathrm{x}$ & & $\mathrm{X}$ & & $\mathrm{X}$ & $\mathrm{X}$ & $\mathrm{X}$ & & & $\mathrm{x}$ & & & $\mathrm{X}$ & 11 \\
\hline Colombia (2006) & $\mathrm{x}$ & & $\mathrm{X}$ & $\mathrm{X}$ & $\mathrm{x}$ & $\mathrm{X}$ & $\mathrm{X}$ & & $\mathrm{X}$ & $\mathrm{x}$ & $\mathrm{X}$ & & & $\mathrm{x}$ & & $\mathrm{X}$ & $\mathrm{X}$ & 12 \\
\hline Costa Rica (2011) & & $\mathrm{X}$ & $\mathrm{x}$ & $\mathrm{X}$ & & & $\mathrm{X}$ & & & $\mathrm{X}$ & $\mathrm{X}$ & & & $\mathrm{x}$ & $\mathrm{X}$ & $\mathrm{X}$ & $\mathrm{x}$ & 10 \\
\hline Cuba (2013) & & & $\mathrm{X}$ & $\mathrm{X}$ & & & & & $\mathrm{X}$ & $\mathrm{X}$ & & & & $\mathrm{X}$ & & $\mathrm{X}$ & $\mathrm{X}$ & 7 \\
\hline Ecuador (1980) & & & & $\mathrm{X}$ & $\mathrm{x}$ & $\mathrm{x}$ & $\mathrm{X}$ & & $\mathrm{X}$ & $\mathrm{X}$ & & & & $\mathrm{x}$ & & $\mathrm{X}$ & $\mathrm{x}$ & 9 \\
\hline $\begin{array}{l}\text { El Salvador } \\
(2012)\end{array}$ & $\mathrm{X}$ & & & $\mathrm{X}$ & $\mathrm{X}$ & $\mathrm{X}$ & $\mathrm{X}$ & & $\mathrm{X}$ & $\mathrm{X}$ & & & & $\mathrm{X}$ & & $\mathrm{X}$ & $\mathrm{X}$ & 10 \\
\hline Guatemala (2000) & & & $\mathrm{x}$ & $\mathrm{X}$ & $\mathrm{X}$ & & $\mathrm{X}$ & & & & $\mathrm{X}$ & & & $\mathrm{x}$ & $\mathrm{X}$ & & $\mathrm{X}$ & 8 \\
\hline Honduras (1979) & & & $\mathrm{x}$ & $\mathrm{x}$ & $\mathrm{x}$ & & $\mathrm{X}$ & & & & & & & $\mathrm{x}$ & & & $\mathrm{X}$ & 6 \\
\hline México (1996) & & & & $\mathrm{x}$ & $\mathrm{x}$ & & $\mathrm{X}$ & & & & & & & $\mathrm{X}$ & & $\mathrm{X}$ & & 5 \\
\hline Panamá (2007) & $\mathrm{X}$ & & $\mathrm{X}$ & $\mathrm{X}$ & $\mathrm{X}$ & $\mathrm{X}$ & $\mathrm{X}$ & & & & & & & $\mathrm{X}$ & & $\mathrm{X}$ & & 8 \\
\hline Paraguay (1999) & $\mathrm{X}$ & & $\mathrm{X}$ & $\mathrm{x}$ & $\mathrm{X}$ & & & & & & $\mathrm{X}$ & & & $\mathrm{x}$ & & $\mathrm{X}$ & & 7 \\
\hline Perú (1988) & & & & $\mathrm{x}$ & $\mathrm{x}$ & & & & & $\mathrm{x}$ & & & & $\mathrm{x}$ & & & $\mathrm{X}$ & 5 \\
\hline $\begin{array}{c}\text { República } \\
\text { Dominicana } \\
(1994)\end{array}$ & $\mathrm{X}$ & & $\mathrm{X}$ & $\mathrm{X}$ & $\mathrm{X}$ & & $\mathrm{X}$ & & $\mathrm{X}$ & & & & & $\mathrm{X}$ & $\mathrm{X}$ & $X$ & $\mathrm{X}$ & 10 \\
\hline Uruguay (2013) & $\mathrm{X}$ & $\mathrm{x}$ & $\mathrm{x}$ & $\mathrm{x}$ & & $\mathrm{x}$ & $\mathrm{X}$ & & $\mathrm{X}$ & $\mathrm{x}$ & $\mathrm{X}$ & & & $\mathrm{x}$ & $\mathrm{X}$ & $\mathrm{X}$ & $\mathrm{X}$ & 13 \\
\hline Venezuela (2013) & & & & $\mathrm{x}$ & $\mathrm{x}$ & $\mathrm{X}$ & $\mathrm{X}$ & & $\mathrm{X}$ & $\mathrm{X}$ & & & & $\mathrm{x}$ & & $\mathrm{X}$ & & 8 \\
\hline
\end{tabular}

* Entre paréntesis aparece el año de creación/modificación del código.

Fuente: elaboración propia. 


\section{Objetivo periodístico}

Todos los códigos analizados contemplan referencias a este marco, pero las más numerosas y relevantes desde el punto de vista del periodismo digital son las que tienen que ver con el servicio público. Destaca especialmente el código de Costa Rica. Su artículo 19 señala que es obligación de todos los miembros "aprovechar al máximo las Nuevas Tecnologías de la Información y la Comunicación —NTc—, para el bien común”.

Mientras el principio de objetividad aparece, por ejemplo, en el artículo 1 del código ecuatoriano: "El periodista tiene la obligación de proporcionar a la comunidad información objetiva, veraz y oportuna”. Asimismo, la honestidad es otra de las categorías encuadradas en este marco ético. En este caso, destaca el ejemplo del código venezolano, que en su apartado de exposición de motivos indica que "el periodista se debe al público y por ello su información debe contribuir con su imparcialidad, veracidad, oportunidad y honestidad a que la verdad del suceso difundido sea evidente".

\section{Privacidad}

Quince de los dieciocho códigos analizados (83.3\%) contienen principios relacionados con esta cuestión. Ahora bien, su distribución es muy desigual, puesto que todos ellos se refieren al respeto por la privacidad, pero ninguno hace mención alguna a la seguridad de la información.

La redacción concreta de esos principios no hace referencia explícita a internet o el universo digital y se reduce, en la mayoría de los casos, a una proclamación genérica del respeto por la vida privada de las personas. Así sucede, por ejemplo, en el código panameño, el cual en su artículo 5 señala que el periodista respetará "el derecho a la intimidad, honra y fama, de las personas públicas y privadas”. Cabe señalar la redacción más detallada del artículo 5 del código de Venezuela: "El periodista solo podrá informar, de la vida privada, aquello que sea de importancia para los intereses de la colectividad; está obligado a darles el tratamiento ajustado a la dignidad, la discreción y la veracidad que se merece la vida privada de cualquier ciudadano venezolano". 


\section{Verificación}

Los principios relacionados con este frame están presentes en 14 de los 18 códigos analizados $(77.8 \%)$. En lo que se refiere a la categoría de confirmación y análisis de datos, destaca, por ejemplo, el código de El Salvador, que en su artículo 31 indica que "en el caso de información obtenida mediante internet, se deberá comprobar la legitimidad de la procedencia del sitio web”.

Evitar la manipulación de los datos es otra categoría de este marco, la cual aparece en la mitad de los códigos analizados, como el de Ecuador, que establece en su apartado introductorio que el periodista "deberá ser ajeno a [... cualquier tipo de manipuleo de la información o de la opinión que falsee, tergiverse, niegue o limite la verdad".

Por lo que respecta a la contextualización de los datos, destaca la referencia del código de Chile en su artículo 7, que señala que "en el caso del periodismo digital, debiera además, incluir un link o un vínculo a la fuente, a fin de ampliar la información para el lector o lectora, en caso de que lo necesite".

\section{Consecuencias}

Todos los códigos contienen menciones a cuestiones relacionadas con este marco que, de hecho, es el más presente en los documentos analizados. Con respecto a la protección de las fuentes, el código peruano, por ejemplo, en su artículo 9, indica que "los periodistas mantendrán en secreto las fuentes de sus fuentes de información”, mientras que el paraguayo señala en su artículo 4: "Todo periodista tiene el deber y el derecho de guardar el secreto profesional, respetando las fuentes de información y las confidencias y no revelando los nombres de sus fuentes ni nada que ellas prohíban comunicar".

El código de Uruguay, por su parte, en su artículo 50, contempla la interactividad con el público de la siguiente manera: "Los periodistas deben promover la permanente capacitación en nuevas tecnologías de la información, a fin de lograr una mejorar interacción con las audiencias y al mismo tiempo una discusión sobre los dilemas éticos de su utilización”. Otro principio que goza de especial interés en los códigos es la autoría de los datos 
y los resultados. El artículo 31 del código de El Salvador establece la necesidad de "respetar y reconocer la autoría intelectual de la información que se publique y consigue mediante internet y otros medios de información".

Por su parte, el código de República Dominicana señala en su artículo 20 que "el periodista estará obligado a respetar el derecho de autoría y, en consecuencia, a citar las fuentes bibliográficas", y añade, en el artículo 41, que "el periodista no deberá presentar como propio ningún material informativo o programa, en cuya preparación no haya prestado su concurso activamente".

Respecto del principio de adecuación a las normas legales, el código hondureño, en su artículo 2, obliga a que los periodistas colegiados cumplan "con las disposiciones de este Código, Ley Orgánica del Colegio de Periodistas de Honduras, y sus Reglamentos, Ley de Emisión del Pensamiento, demás Leyes de la República y Resoluciones legalmente emanadas del Colegio".

\section{Conclusiones}

La primera conclusión que se puede extraer es que la presencia de los principios relativos al big data en los códigos analizados es muy desigual, con países cuyas normas deontológicas de referencia incluyen más de la mitad de esos principios, como Uruguay, Colombia, Chile, Costa Rica y Ecuador, y otros que recogen menos de un tercio, como México o Perú. Del mismo modo, es una presencia incompleta, por cuanto tres principios muy importantes en este contexto, como son el control del software y las técnicas empleadas, la fiabilidad de los enlaces y los hipervínculos y la seguridad de la información, no son mencionados en ninguno de los códigos analizados (tabla 2).

Asimismo, el año de la aprobación o de la última modificación de los códigos es un factor importante que determina su contenido, en general respecto de una mayor adaptación al periodismo desarrollado en la era del big data, pero no constituye una garantía absoluta en ese sentido. Así, por ejemplo, si bien los códigos de los tres países con mayor presencia de 
principios de ese tipo (Uruguay, Colombia o Chile) han sido aprobados o modificados en los últimos años, se observan otros países con documentos también recientes, como Argentina o Cuba, cuya adaptación al contexto del big data es sensiblemente menor (tabla 3). De hecho, los países con códigos aprobados en 1994 (República Dominicana) o incluso en 1980 (Ecuador) recogen un mayor número de normas aplicables a este tipo de periodismo que los códigos argentino o cubano.

En cuanto a los frames y normas concretas con mayor presencia, llama la atención que cuestiones relativas al objetivo periodístico, como la objetividad, la honestidad o el servicio público, que constituyen principios éticos clásicos para el periodismo general, sean los más presentes en los códigos. En cambio, la verificación de los datos, frame mucho más vinculado a las técnicas del big data y que hace referencia a asuntos fundamentales en el periodismo digital, como la contextualización de los datos o la fiabilidad de los enlaces, solo la recoge $27.8 \%$ de los códigos estudiados.

En ese sentido, como señala el Comité de Ética y Valores de la American Society of News Editors (citado en Mitchell, 2002), las reglas éticas tradicionales también se aplican en línea, pero al mismo tiempo es evidente que algunas de ellas adquieren una nueva dimensión en el entorno virtual. Sirvan como ejemplo, por citar dos cuestiones muy relacionadas, el big data y el periodismo, la privacidad o el tratamiento de los datos. Si bien se trata de cuestiones recogidas por los códigos, la redacción es en la mayoría de los casos muy genérica, por lo cual pueden surgir situaciones en el día a día para los que no ofrezcan una respuesta adecuada para el periodista.

Asimismo, una de las posibles recomendaciones que pueden derivarse de este estudio consiste en la promulgación de códigos o, en su defecto, documentos con recomendaciones, que recojan de manera específica directrices relativas al big data, a semejanza de lo que está sucediendo en los últimos años en varios países. Así ha sucedido con la cobertura de catástrofes naturales o atentados terroristas (numerosas asociaciones profesionales han redactado documentos éticos monográficos sobre estas cuestiones tras los atentados del 11-S), con el tratamiento periodístico de la infancia 
y la juventud (recomendaciones en esa línea también han proliferado, sobre todo, por el auge de contenidos sensacionalistas en las televisiones), $\mathrm{o}$ más recientemente y en relación con el periodismo digital, con la presencia de los periodistas en las redes sociales.

De la misma manera, sería recomendable una revisión a fondo de aquellos códigos, cuya última renovación data de hace varias décadas, y que en esa nueva redacción se incorpore un apartado específico sobre el periodismo digital, como ya ocurre con los de Chile o Uruguay, de manera que se dé una respuesta lo más adecuada y actualizada posible a los dilemas éticos que día a día surgen en relación con estas cuestiones.

En la misma línea, cabe recordar que el código supranacional que sirve como referencia en el ámbito geográfico estudiado (el Código Latinoamericano de Ética Periodística) data de 1979. Una revisión de este texto no solo serviría para actualizar sus contenidos, sino también, en la medida en que fue promulgado por una entidad en la que están representadas las organizaciones profesionales de la mayoría de los países de esa región, para realizar una puesta en común que resultaría muy útil para cada uno de esos colectivos profesionales. Parece razonable también que la formación de un hipotético equipo de trabajo para llevar a cabo esa revisión debería combinar a especialistas en periodismo en general con especialistas en periodismo y big data para que estos últimos aportaran su perspectiva en esas cuestiones.

En estas revisiones, no solo sería conveniente dar cabida a cuestiones específicas del periodismo de datos que, en algunos casos, no están presentes en ninguno de los códigos, como la fiabilidad de los enlaces o la seguridad de la información, sino que habría que reelaborar esas otras cuestiones éticas clásicas que en el periodismo digital adquieren una nueva dimensión.

Por último, este estudio plantea la realización posterior, por un lado, de un análisis similar de los códigos de otras regiones del mundo, para poder comparar los resultados; y por otro, de un análisis de las directrices promulgadas por medios de comunicación o empresas periodísticas concretas en el mismo ámbito de América Latina. 


\section{Referencias}

Anderson, C. (2008). The end of theory: The data deluge makes the scientific method obsolete. Wired Magazine, 16(7). Recuperado de https://www.wired.com/2008/06/pb-theory/

Anderson, C. W. (2012). Towards a sociology of computational and algorithmic journalism. New Media and Society, 15(7), 1005-1021. doi: $10.1177 / 1461444812465137$

Associated Press (31 mayo 2017). Data journalism chapter debuts in 2017 AP Stylebook. Recuperado de https://www.ap.org/press-releases/2017/data-journalism-chapter-debuts-in-2017-ap-stylebook

Aznar, H. (2005). Ética de la comunicación y nuevos retos sociales: códigos y recomendaciones para los medios. Barcelona: Paidós.

Aznar, H. (2011). Comunicación responsable: deontología y autorregulación de los medios. Barcelona: Ariel.

Boyd, D. y Crawford, K. (2012). Critical questions for big data: Provocations for a cultural, technological, and scholarly phenomenon. Information, Communication \& Society, 15, 662-679. doi: 10.1080/1369118X.2012.678878.

Bradshaw, P. (2014). Data journalism. En L. Zion y D. Craig (eds.), Ethics for digital journalists: Emerging best practices (pp. 202-219). Nueva York: Routledge.

Bruns, A. (2016). Big data analysis. En T. Witschge, C. W. Anderson, D. Domingo y A. Hermida (eds.), The Sage handbook of digital journalism (pp. 509-527). Thousand Oaks, CA: SAGE.

Carlson, M. (2015). The robotic reporter: Automated journalism and the redefinition of labor, compositional forms, and journalistic autho- 
rity. Digital Journalism, 3(3), 416-431. doi:10.1080/21670811.2 014.976412

Casero-Ripollés, A. (2012). Contenidos periodísticos y nuevos modelos de negocio: evaluación de servicios digitales. El Profesional de la Información, 21(4), 341-346. doi: 10.3145epi.2012.jul.02

Chaparro, M.Á. (2014). Nuevas formas informativas: el periodismo de datos y su enseñanza en el contexto universitario. Historia y Comunicación Social, 19, 43-54.doi: 10.5209/rev_HICS.2014.v19.45009

Coddington, M. (2015). Clarifying journalism's quantitative turn: A typology for evaluating data journalism, computational journalism, and computer-assisted reporting. Digital Journalism, 3(3), 331348. doi: 10.1080/21670811.2014.976400

Craig, D., Ketterer, S. y Yousuf, M. (2017). To post or not to post: Online discussion of gun permit mapping and the development of ethical standards in data journalism. Journalism \& Mass Communication Quarterly, 94(1), 168-188. doi: 10.1177\%2F1077699016684796

Crawford, K., Miltner, K. y Gray, M. L. (2014). Critiquing big data: Politics, ethics, epistemology. International Journal of Communication, 8, 1663-1672. Recuperado de http://ijoc.org/index.php/ijoc/ article/view/2167/1164

Crucianelli, S. (2012). Introducción al periodismo de datos. Recuperado de https://knightcenter.utexas.edu/mooc/datos/semanal/periodismodedatosintroduccion.pdf

Dader, J. L. (1997). Periodismo de precisión. Madrid: Síntesis.

Deuze, M. y Yeshua, D. (2001). Online journalists face new ethical dilemmas: Lessons from the Netherlands. Journal of Mass Media Ethics, 16(4), 273-292. doi: 10.1207/S15327728JMME1604_03 
Diakopoulos, N. (2015). Algorithmic accountability. Digital Journalism, 3(3), 398-415. doi: 10.1080/21670811.2014.976411

Díaz-Campo, J. (2004). Códigos éticos del periodismo latinoamericano. En L. H. Marcos (coord.), O livro de atas do XIII IBERCOM (pp. 516-524). Santiago de Compostela: Universidad de Santiago de Compostela.

Díaz-Campo, J. (2016). Consideraciones éticas sobre el contenido generado por el usuario en el periodismo digital. En J. C. Suárez Villegas y J. Cruz Álvarez (eds.), Desafíos éticos en el periodismo digital (pp. 71-92). Madrid: Dykinson/Ministerio de Economía y Competitividad.

Díaz-Campo, J., Chaparro-Domínguez, M.Á. y Segado-Boj, F. (2016).Ética y periodismo de datos en los códigos de los medios españoles. En E. Blanco Castilla y M. Quesada (eds.), Periodismo de datos (pp. 159-186). La Laguna: Cuadernos Artesanos de Comunicación Latina, 112.

Díaz-Campo, J. y Segado-Boj, F. (2014). La adaptación de los códigos de ética periodística europeos a internet y las TIC. Ámbitos: Revista Internacional de Comunicación, 26. Recuperado de https://hal. archives-ouvertes.fr/hal-01343850

Díaz-Campo, J. y Segado-Boj, F. (2015). Journalism ethics in a digital environment: How journalistic codes of ethics have been adapted to the internet and ICTs in countries around the world. Telematics and Informatics, 32(4), 735-744. doi:10.1016/j.tele.2015.03.004

Dörr, K. N. y Hollnbuchner, K. (2017). Ethical challenges of algorithmic journalism. Digital Journalism, 5(4), 404-419. doi: $10.1080 / 21670811.2016 .1167612$

Elías-Pérez, C. (2015). Big data y periodismo en la sociedad red. Madrid: Síntesis. 
Ess, C. (2009). Digital media ethics. Londres: Polity Press.

Fairfield, J. y Shtein, H. (2014). Big data, big problems: Emerging issues in the ethics of data science and journalism. Journal of Mass Media Ethics, 29(1), 38-51. doi: 10.1080/08900523.2014.863126

Ferreras, E. (2013). Aproximación teórica al perfil profesional del periodista de datos. Icono 14, 11(2), 115-140. doi: 10.7195/ri14.v11i2.573.

Fink, K. y Anderson, C. W. (2015). Data journalism in the United States: Beyond the "usual suspects". Journalism Studies, 16(4), 467-481. doi: 10.1080/1461670X.2014.939852

García-Avilés, J. A. (2014). Online newsrooms as communities of practice: Exploring digital journalists' applied ethics. Journal of Mass Media Ethics, 29(4), 258-272. doi: 10.1080/08900523.2014.946600

Gray, J., Chambers, L. y Bounegru, L. (eds.) (2012). The data journalism handbook: how journalists can use data to improve the news. Cambridge: O’Reilly.

Hayes, A. S., Singer, J. B. y Ceppos, J. (2007). Shifting roles, enduring values: The credible journalist in a digital age. Journal of Mass Media Ethics, 22(4), 262-279. doi: 10.1080/08900520701583545

Kent, T. (2015). An ethical checklist for robot journalism. Recuperado de https://medium.com/@tjrkent/an-ethical-checklist-forrobotjournalism-1f41dcbd7be2

La-Rosa, L. y Sandoval-Martín, T. (2016). La insuficiencia de la Ley de Transparencia para el ejercicio del Periodismo de datos en España. Revista Latina de Comunicación Social, 71, 1208-1229. doi: 10.4185/RLCS-2016-1142

Lewis, S. C. y Westlund, O. (2015). Big data and journalism. Digital Journalism, 3(3), 447-466. doi: 10.1080/21670811.2014.976418 
López-García, X., Toural-Bran, C. y Rodríguez-Vázquez, A. I. (2016). Software, estadística y gestión de bases de datos en el perfil del periodista de datos. El Profesional de la Información, 25(2), 286-294. doi: 10.3145/epi.2016.mar.16

López-Talavera, M. M. (1998). Fundamentos éticos de la prensa en América Latina (Tesis doctoral, Universidad Complutense de Madrid, España).

Mayer-Schönberger, V. y Cukier, K. (2013). Big data: A revolution that will transform how we live, work, and think. Boston: Houghton Mifflin Harcourt.

McBride, R.E.D. (2016).The ethics of data journalism.DigitalCommons@ University of Nebraska - Lincoln, 1-44. Recuperado de http://digitalcommons.unl.edu/journalismprojects/9/

Mitchell, B. (12 agosto 2002). "New media" bring a new set of problems. En Poynter. Recuperado de https://www.poynter.org/news/newmedia-bring-new-set-problems

Oller, M. y Barredo, D. (2013). Las culturas periodísticas intermedias: estudios comparativos internacionales en periodismo. La Laguna: Cuadernos Artesanos Latina, 35.

Parasie, S. (2015). Data-driven revelation? Digital Journalism, 3(3), 364380. doi: $10.1080 / 21670811.2014 .976408$

Parra Valcarce, D., Real Rodríguez, E. y López Talavera, M. M. (2017). Códigos éticos en el ciberperiodismo español y latinoamericano. Naveg@mérica, 18. Recuperado de http://revistas.um.es/navegamerica/article/view/286541

Pavlik, J. (2001). Journalism and new media. Nueva York: Columbia University Press. 
Perry, F. y Paz, M. (eds.) (2014). Manual de periodismo de datos iberoamericano. Fundación Poderomedia y Escuela de Periodismo de la Universidad Alberto Hurtado. Recuperado de http://manual.periodismodedatos.org/index.php

Singer, J. B. (2007). Contested autonomy: Professional and popular claims on journalistic norms. Journalism Studies, 8(1), 79-95. doi: $10.1080 / 14616700601056866$

Spones (19 enero 2013). Dwight R. Worley - The Gun Owner Next Door. Recuperado de http://www.snopes.com/politics/guns/gunmap.asp

Suárez-Gonzalo, S. (2017). Big social data: límites del modelo notice and choice para la protección de la privacidad. El Profesional de la Información, 26(2), 283-292. doi: 10.3145/epi.2017.mar.15

Suárez Villegas, J. C. (1999). Medios de comunicación y autocontrol: entre la ética y el derecho. Sevilla: MAD.

Suárez Villegas, J. C. (2015). Nuevas tecnologías y deontología periodística: comparación entre medios tradicionales y nativos digitales. El Profesional de la Información, 24(4), 390-395. doi: 10.3145/ epi.2015.jul.05

Ward, S. J. A. (2010). Global journalism ethics. Montreal: McGill-Queen's University Press. 


\section{Anexo: códigos deontológicos analizados}

Asociación de Entidades Periodísticas Argentinas: Code of Ethics of the Argentine Press. Recuperado de https://accountablejournalism. org/ethics-codes/asociacion-de-entidades-periodisticas-argentinas-code-of-ethics-of-the-arge

Código Nacional de Ética Periodística de Bolivia. Recuperado de https:// www.bolpress.com/2016/09/29/codigo-nacional-de-etica-periodistica-de-bolivia/

Código de Ética e Autorregulamentação, Code of Ethics for the Association of National Journalists (Brazil). Recuperado de https://accountablejournalism.org/ethics-codes/association-of-national-journalists-brazil-code-of-ethics

Código de Ética del Colegio de Periodistas de Chile. Recuperado de http:// www.colegiodeperiodistas.cl/p/etica-periodistica.html

Conozca el Código de Ética del Círculo de Periodistas de Bogotá. Recuperado de http:/ / eticasegura.fnpi.org/2011/12/27/conozca-el-codigo-de-etica-del-circulo-de-periodistas-de-bogota/

Código de Ética del Colegio dePeriodistas de Costa Rica. Recuperado de http:// www.colper.or.cr/userfiles/file/juridico/codigos/codigo_etica.pdf

Código de Ética del Periodista de Cuba. Recuperado de http://www.cubaperiodistas.cu/index.php/codigo-de-etica-del-periodista/

Código de Ética Periodística en el Ecuador. Recuperado de https://eticadelacomunicacion.wordpress.com/2008/06/18/codigo-de-etica-periodistica-en-el-ecuador/

Conozca el Código Ético de los periodistas de El Salvador. Recuperado de http://eticasegura.fnpi.org/2016/09/08/conozca-codigo-eticolos-periodistas-salvador/ 
Code of Ethics of the Association of Journalists of Guatemala. Recuperado de https://accountablejournalism.org/ethics-codes/Guatemala-Declaration

Código deética del Colegio dePeriodistas deHonduras. Recuperadodehttp:// colegiodeperiodistasdehonduras.hn/l/?q=es/c \%C3 \%B3digode- \%C3 \%A9tica-del-colegio-de-periodistas-de-honduras

Mexico: Journalists' Code of Ethics. Recuperado de https://accountablejournalism.org/ethics-codes/Mexico-Journalist

Código ético del Forum de Periodistas de Panamá. Recuperado de http:// eticasegura.fnpi.org/2016/09/22/codigo-etico-del-forum-periodistas-panama/

Código de Ética del Sindicato de Periodistas del Paraguay. Recuperado de http://www.ultimahora.com/hay-un-codigo-etica-que-necesitadesempolvarse-n650857.html

Code of Ethics of the National Association of Journalists of Peru. Recuperado dehttps://accountablejournalism.org/ethics-codes/Peru-ANP

Código de Ética del Periodista Dominicano. Recuperado de http://www. unesco.org/fileadmin/MULTIMEDIA/HQ/CI/CI/images/ wmn/code \%20of \%20Ethics \%20Collegio \%20de \%20periodistas.pdf

Código de Ética Periodística de Uruguay. Recuperado de http://www. apu.org.uy/wp-content/uploads/2013/04/C \%C3 \%B3digode- \%C3 \%A9tica-period \%C3 \%ADstica-Versi \%C3 \%B3n-Final-9-4-13.pdf

Código de Ética del Periodista Venezolano. Recuperado de http://cdn1. cnpven.org/archivos/80/original_codigoeticafinal.pdf 